\title{
Prognostic Factors in Recurrent Congenital Muscular Torticollis
}

\author{
Chotigavanichaya $C, M D$, Phongprapapan $\mathbf{P}, M D$, Wongcharoenwatana J, MD, Eamsobhana $\mathbf{P}, \mathrm{MD}$, \\ Ariyawatkul T, MD, Kaewpornsawan K, MD,
}

Department of Orthopaedic Surgery, Faculty of Medicine Siriraj Hospital, Bangkok, Thailand

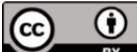 \\ This is an open-access article distributed under the terms of the Creative Commons Attribution License, which permits unrestricted use, \\ distribution, and reproduction in any medium, provided the original work is properly cited \\ Date of submission: 20th January 2020 \\ Date of acceptance: 02nd February 2021
}

\begin{abstract}
Introduction: Congenital muscular torticollis (CMT), primarily resulting from unilateral shortening and fibrosis of the sternocleidomastoid muscle. One of the common surgical complications is recurrent deformity. However, the associations between unipolar or bipolar release, age of the patient, and the recurrence of the disease are unclear. Therefore, the purpose of this study was to evaluate the factors associated with recurrence after surgery.

Materials and Methods: A retrospective review was performed in 47 patients who were diagnosed with CMT and had been treated surgically with unipolar or bipolar release between January 2007 and December 2015. Demographic data (sex, sides, surgical technique, age at time of surgery, period of follow-up, complications and recurrence) were recorded.

Results: Forty-seven patients with an average age of 8.7 years old at time of surgery. Twenty-six patients had rightsided muscular torticollis, while 21 had left-sided. The average follow-up time was 2 years (range, $2-4$ years). The average age of unipolar release was 8.8 years old (range, 218 years old), while the average age of bipolar release was 8.7 years old (range, 2-13 years old). Recurrence occurred in 11 patients ( 9 in unipolar and 2 in bipolar release). Sex, side of deformity, type of surgery and age at time of surgery showed no statistically significant as a factor for recurrence rate, however recurrence of unipolar more than bipolar surgery was nearly two times revealing clinical significance. Conclusions: Sex, side of deformity, type of surgery and age at time of surgery were not associated with the recurrence deformity.
\end{abstract}

Keywords: congenital muscular torticollis, unipolar release, bipolar release, recurrence deformity

\section{INTRODUCTION}

Congenital muscular torticollis was the most common neck problem in children. The incidence was 1:300 live births ${ }^{1}$. Clinical evidence of torticollis presented initially as a palpable mass or tightness at the involved side; this may be a pseudotumor of the sternocleidomastoid muscle, which causes the cock robin posture 2 .

Etiology of this disease was still unknown ${ }^{3}$. However, previous literatures suggested causes are compartment syndrome during perinatal period from soft tissue compression of the neck at the time of delivery ${ }^{4}$; intrauterine crowding, based on the high association with breech presentation and developmental dysplasia of the hip ${ }^{5}$; primary neurogenic cause, histopathologic denervation and reinnervation due to a traumatic event ${ }^{6}$ (ischemia) and develop fibrosis of the sternocleidomastoid muscle; and the mesenchymal theory ${ }^{7}$, which is related to an environmental change, resulting in mesenchymal cells de-differentiating.

Initial treatment started with non-surgical intervention. Physical therapy program including stretching exercises ${ }^{8}$ showed excellent result in $90 \%$ of cases, especially in younger patients. Surgical treatment was recommended for patients who had persistent torticollis after 1-4 years of age?. Choices for surgical treatment were unipolar release or bipolar release. Recurrence was one of common complications after surgical release for torticollis. Shim JS et al reported proper time of surgery was the most important factor influences due to more prognosis was the proper time of surgery, that mean the patients can cooperate in bracing and rehabilitation program ${ }^{10}$. Chin En Chen and Jih Yang Ko found recurrence in 1 out of 18 torticollis patients ${ }^{11}$. The influences of sex, sides, surgical technique, and early versus delayed surgical release, on the torticollis recurrence rate remained unclear ${ }^{12,13}$. The purpose of this study was to

Corresponding Author: Chatupon Chotigavanichaya, Department of Orthopaedic Surgery, Mahidol University Faculty of Medicine Siriraj Hospital, 2 Thanon Wang Lang, Siri Rat, Bangkok Noi, Bangkok 10700, Thailand

Email: chatuponc@gmail.com 
evaluate the factors associated with recurrence of congenital muscular torticollis after surgery.

\section{MATERIAL AND METHOD}

A retrospective review was conducted on 47 congenital muscular torticollis patients who had tightness of the sternocleidomastoid muscle without an apparent mass. Aged between 2-15 years old. All cases were undergone surgical release between January 2007 and December 2015 and were followed-up at two, four and six weeks post-operative then every three months for two years and after that every six months. All cases had at least two years follow-up. The postoperative protocol included soft cervical collar for three weeks then physical therapy for 3-4 weeks. Demographic data (sex, sides of deformity, surgical technique, the age at time of surgery, the follow-up period, complications and recurrence) were recorded. Patients with secondary causes of muscular torticollis such as vertebral anomalies, unilateral atlantooccipital fusion, Klippel-Feil syndrome and unilateral absence of sternocleidomastoid were excluded. Patients with incomplete medical data were also excluded. The definition for recurrence was prominent appearance of sternocleidomastoid muscle with head tilt and 50\% decreased in range of motions compare with first 12 weeks after surgery. Cox regression analysis was used to compare any of the associated prognostic factors and disease recurrence.

\section{RESULTS}

Forty-seven patients were included in the study (Table I and II). The average age at time of surgery was 8.7 years old. Twenty-six patients $(55.3 \%)$ had right-sided muscular torticollis, and twenty-one (44.7\%) exhibited it on the left. Thirty-four patients $(72.3 \%)$ had unipolar release, while 13 $(27.7 \%)$ had bipolar release. The average follow-up time was 33.4 months. Recurrence of the deformity was found in 11 (23.4\%) out of 47 patients; the mean recurrence time was 52.5 months (median: 52.8 months).

There was no statistically significant difference between recurrence of torticollis and the age at time of surgery $(\mathrm{p}=0.467)$, side of the torticollis $(\mathrm{p}=0.157)$ and gender $(p=0.249)$. Moreover, there was no statistically significant difference between the risk of recurrence and technique of surgical release $(\mathrm{p}=0.428)$. From the Kaplan-Meier survival analysis (Fig. 1), first recurrence case developed at 6.1 months, and the patient with the longest follow-up time was a recurrence case developed in 52.8 months.

\section{DISCUSSION}

Congenital muscular torticollis was a common paediatric orthopedic problem. It may be associated with dysplasia of the hip or metatarsus adductus. Some theories suggested that intrauterine molding during pregnancy may cause this deformity ${ }^{14}$. Differential diagnoses of torticollis were basilar impression, C1-2 instability and neurologic torticollis.

The treatment of congenital muscular torticollis initially involved stretching exercises during the first year of age ${ }^{8}$. If patient's neck remained tilted after one year of age, operative treatment was performed. Chandler ${ }^{9}$ advocated that good results can be achieved by performing the surgical procedure between one and four years of age.

In this study, there was nine cases $(9 / 34)$ of recurrence after unipolar release and two cases (2/13) of recurrence after bipolar release. Even though there was no statistically significant between unipolar and bipolar release on recurrence rate, there was clinically significant difference when compared percentage of recurrence between two techniques $(26.5 \%$ in unipolar release and $15.4 \%$ in bipolar release).

Previous literatures showed no recurrence case in patient presenting with CMT after 10 years old who were treated with bipolar release ${ }^{15}$. However, there was still controversy about which type of surgical treatment, unipolar or bipolar, provided better result. Chen et $a l^{11}$ reported a recurrence rate of 1 out of 18 patients (5.5\%) from surgical treatment in congenital muscular torticollis patients aged over six years old. As for the long term results after open surgical tenotomy of the sternal and clavicular origins of the sternocleidomastoid muscle for idiopathic muscular torticollis, Ippolito et $a l^{13}$ reported that the factors affecting the treatment results were patient's age at operation, the disease duration, and severity of the deformity before surgery.

Chen et $a l^{11}$ and Minamitani et $a l^{16}$ reported that excellent results in patients over six years of age and the degree of correction were affected by the adequacy of the surgical treatment and rehabilitation, rather than age. In this study, we evaluated the factors that may affect the post-operative recurrence of torticollis. However, no statistically significant difference was found between the recurrence of the deformity and sex, sides, age at time of surgical treatment and the type of surgery.

However, numerous studies have advocated that age at the of surgical treatment was a risk factor. For instance, it has been recommended that the ideal age for surgery should be between one and four years old ${ }^{9}$. Coventry et $a l^{17}$ suggested that good results can still be achieved at an upper age limit of 12-14 years. Lee et al ${ }^{18}$ suggested that patients between 6 and 16 years had good operative results, and concluded that the patient's age at surgery seemed to be the most important factor. In contrast, Shim and Jang ${ }^{10}$ suggested that age was not the most important factor when determining the optimal 
Table I: Demographic data

\begin{tabular}{|lc|}
\hline Patients characteristics & $\begin{array}{c}\text { Mean } \pm \text { SD / n (\%) } \\
(\mathbf{n}=47)\end{array}$ \\
\hline Age (yrs) & $8.7 \pm 3.7$ \\
Gender & $9(2-18)^{*}$ \\
Male & $20(42.6 \%)$ \\
Female & $27(57.4 \%)$ \\
Affected side & $21(44.7 \%)$ \\
Left & $26(55.3 \%)$ \\
Right & $34(72.3 \%)$ \\
Surgical procedure & $13(27.7 \%)$ \\
Unipolar & $11(23.4 \%)$ \\
Bipolar & $36(76.6 \%)$ \\
Recurrence & $11(23.4 \%)$ \\
Yes & $8(17.0 \%)$ \\
No & $16(34.0 \%)$ \\
Lee Score & $12(25.5 \%)$ \\
Poor & $33.4 \pm 12.0$ \\
Fair & $31.4(6.1-70.1)^{*}$ \\
Good & \\
Excellent & \\
Follow-up time (months) & \\
& \\
\hline
\end{tabular}

*Median (Min-Max)

Table II: Clinical Outcome

\begin{tabular}{|lcccc|}
\hline Factors & $\begin{array}{c}\text { Recurrence } \\
(\mathbf{n}=\mathbf{1 1})\end{array}$ & $\begin{array}{c}\text { Non-recurrence } \\
(\mathbf{n = 3 6 )}\end{array}$ & $\begin{array}{c}\text { Univariate } \\
\text { Hazard ratio }\end{array}$ & p value \\
\hline $\begin{array}{l}\text { Age at surgery (yrs.) } \\
\text { Gender }\end{array}$ & $9.5 \pm 4.5$ & $8.5 \pm 3.5$ & $1.07(0.89,1.29)$ & 0.467 \\
$\quad$ Male (ref) & $3(27.3 \%)$ & $17(47.2 \%)$ & 2.39 & 0.249 \\
$\quad$ Female & $8(72.7 \%)$ & $19(52.8 \%)$ & $(0.54,10.48)$ & 0.157 \\
$\begin{array}{l}\text { Affected side } \\
\quad \text { Right (ref) }\end{array}$ & $4(36.4 \%)$ & $22(61.1 \%)$ & 2.75 & 0.428 \\
$\quad$ Left & $7(63.6 \%)$ & $14(38.9 \%)$ & $(0.68,11.14)$ & \\
Surgical procedure & & & 1.98 \\
$\quad$ Unipolar & $9(81.8 \%)$ & $25(69.4 \%)$ & $(0.37,10.72)$ & \\
$\quad$ Bipolar (ref) & $2(18.2 \%)$ & $11(30.6 \%)$ & & \\
\end{tabular}

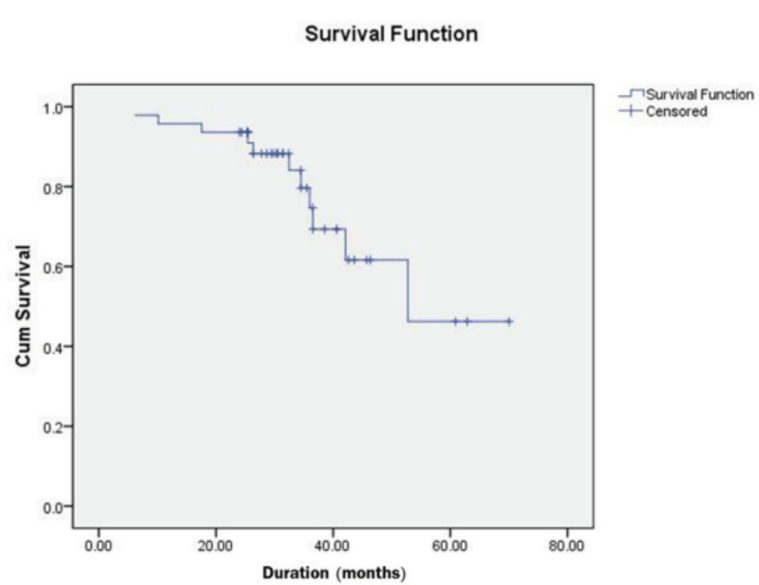

Fig. 1: Survival curve for all congenital muscular torticollis patients. The first case of recurrence $(1 / 11)$ was occurred at 6.1 months and last case of recurrence (11/11) was occurred at 52.8 months post-operative. time for the operation, but compliance with a post-operative rehabilitation program was the most important consideration. They recommended that congenital muscular torticollis surgery should be delayed until the patient's compliance with the planned rehabilitation can be guaranteed.

In this study, recurrences occurred in 11 patients (23.4\%), but they were not related to the age at time of surgery or the surgery type. The recurrences might occur due to the retained fibrous band or fascia of the sternocleidomastoid. As patients grew older, their body weight and height increase, thereby affecting the sternocleidomastoid fascia and vertebral bodies. This can lead to tightness of the fibrous band and resulting in a recurrence of torticollis.

The first case of recurrences developed early at 6.1 months after surgery, while the second case occurred in 10.1 months This suggested that the first follow-up appointment for 
clinical assessment should be scheduled at least 6 months after surgery, and subsequent one every 6-12 months for at least 7 years, to ensure that no recurrence has developed.

The limitations of this study were the small number of patients and the different surgeons may have employed varying decision-making techniques. We were also unable to evaluate the post-operative protocol due to the study's retrospective nature.

\section{CONCLUSION}

It is controversial whether unipolar or bipolar release produces better results. Sex, sides of deformity, type of surgery and age at the time of surgery were not associated with the recurrence of congenital muscular torticollis. Consequently, choices of surgery and the rehabilitation protocols should be discussed comprehensively, and the possibility of recurrence after surgical release should be advised.

\section{CONFLICT OF INTEREST}

The authors declare no potential conflict of interest

\section{REFERENCES}

1. Freed SS, Coulter-O'Berry C. Identification and Treatment of Congenital Muscular Torticollis in Infants. J Prosthet Orthot. 2004; 16(4): S18-S23. doi: 10.1097/00008526-200410001-00007

2. Morrison DL, MacEwen GD. Congenital muscular torticollis: observations regarding clinical findings, associated conditions, and results of treatment. J Pediatr Orthop. 1982; 2(5): 500-5.

3. Shim JS, Noh KC, Park SJ. Treatment of congenital muscular torticollis in patients older than 8 years. J Pediatr Orthop. 2004; 24(6): 683-8. doi: 10.1097/00004694-200411000-00016

4. Davids JR, Wenger DR, Mubarak SJ. Congenital muscular torticollis: sequela of intrauterine or perinatal compartment syndrome. J Pediatr Orthop. 1993; 13(2): 141-7.

5. Ling CM, Low YS. Sternomastoid tumor and muscular torticollis. Clin Orthop Relat Res. 1972; 86: 144-50. doi: 10.1097/00003086-197207000-00020

6. Sarnat HB, Morrissy RT. Idiopathic torticollis: sternocleidomastoid myopathy and accessory neuropathy. Muscle Nerve. 1981; 4(5): 374-80. doi: 10.1002/mus.880040505

7. Tang S, Liu Z, Quan X, Qin J, Zhang D. Sternocleidomastoid pseudotumor of infants and congenital muscular torticollis: finestructure research. J Pediatr Orthop. 1998; 18(2): 214-8.

8. Cheng JC, Wong MW, Tang SP, Chen TM, Shum SL, Wong EM. Clinical determinants of the outcome of manual stretching in the treatment of congenital muscular torticollis in infants. A prospective study of eight hundred and twenty-one cases. $\mathrm{J}$ Bone Joint Surg Am. 2001; 83(5): 679-87. doi: 10.2106/00004623-200105000-00006

9. Chandler FA. Congenital muscular torticollis. Bull Hosp Joint Dis. 1953; 14(2): 158-71.

10. Shim JS, Jang HP. Operative treatment of congenital torticollis. J Bone Joint Surg Br. 2008; 90(7): 934-9. doi: 10.1302/0301620X.90B7.20339

11. Chen CE, Ko JY. Surgical treatment of muscular torticollis for patients above 6 years of age. Arch Orthop Trauma Surg. 2000; 120(3-4): 149-51. doi: 10.1007/s004020050032.

12. Cheng JC, Au AW. Infantile torticollis: a review of 624 cases. J Pediatr Orthop. 1994;14(6):802-8.

13. Ippolito E, Tudisco C, Massobrio M. Long-term results of open sternocleidomastoid tenotomy for idiopathic muscular torticollis. J Bone Joint Surg Am. 1985; 67(1): 30-8.

14. Weiner DS. Congenital dislocation of the hip associated with congenital muscular torticollis. Clin Orthop Relat Res. 1976; (121): 163-5.

15. Sudesh P, Bali K, Mootha AK, Dhillon MS. Results of bipolar release in the treatment of congenital muscular torticolis in patients older than 10 years of age. J Child Orthop. 2010; 4(3): 227-32. doi: 10.1007/s11832-010-0254-x 
16. Minamitani K, Inoue A, Okuno T. Results of surgical treatment of muscular torticollis for patients greater than 6 years of age. J Pediatr Orthop. 1990; 10(6): 754-9. doi: 10.1097/01241398-199011000-00009

17. Coventry MB, Harris LE. Congenital muscular torticollis in infancy; some observations regarding treatment. J Bone Joint Surg Am. 1959; 41(5): 815-22.

18. Lee EH, Kang YK, Bose K. Surgical correction of muscular torticollis in the older child. J Pediatr Orthop. 1986; 6(5): 585-9. doi: 10.1097/01241398-198609000-00011 